\title{
PENSAMIENTO SOCIALCRISTIANO Y CIRCULACIÓN DE LAS IDEAS: REDES A TRAVÉS DE LAS CUALES SE IMPORTARON Y SE EXPORTARON IDEAS DURANTE LOS LARGOS 1960s EN CHILE
}

\author{
Social Christian thought and circulation of ideas: \\ the networks through which ideas were \\ imported and exported in the 1960s in Chile
}

Eduardo Devés-Valdés*

\begin{abstract}
RESUMEN
A la hora de entender la ubicación de la intelectualidad chilena y latinoamericana, en el marco de la circulación mundial de las ideas, estudiar el socialcristianismo de los años 1960s es un caso interesante, tanto por su particular vitalidad, como por ubicarse en la confluencia de varios movimientos. Tratando de entender las síntesis producidas en el socialcristianismo de la época, se apunta a descubrir el proceso de combinación de diferentes líneas de pensamiento, teniendo en cuenta las redes a través de las cuales llegaron y se combinaron. Intentando, por otra parte, no conformarse con la idea normal de quienes estudian la cultura en nuestras regiones, suponiéndonos sólo como importadores, se estudia la exportación de estas síntesis y las redes a través de las cuales ello ocurrió. El trabajo se focaliza en dos casos principalmente: para la importación de ideas, la red "Economía y Humanismo" y para la exportación, la red "Cristianos por el Socialismo".

Palabras-clave: socialcristianismo; pensamiento años 1960s; redes intelectuales; circulación de las ideas; "Economía y Humanismo"; "Cristianos por el Socialismo"; exportaciones eidéticas.
\end{abstract}

de Chile

Investigador del Instituto de Estudios Avanzados (IDEA) de la Universidad de Santiago 


\title{
RESUMO
}

Quando se trata de entender a posição dos intelectuais chilenos e latinoamericanos, no contexto da circulação global de ideias, a pesquisa sobre o cristianismo social na década de 1960 é interessante, porque pode ser localizada na confluência de vários movimentos. Tentar entender as sínteses produzidas no âmbito do cristianismo social da época é descobrir a combinação de diferentes linhas de pensamento, levando em conta as redes através das quais elas chegaram e se combinaram. Tentar, por outro lado, não se conformar com a ideia normal daqueles que estudam a cultura em nossas regiões, digamos, apenas enquanto importadores, possibilita estudar a exportação dessas sínteses e as redes através das quais isso ocorreu. $\mathrm{O}$ trabalho se concentra, principalmente, em dois casos: para a importação de ideias, a rede "Economia e Humanismo" e, para a exportação, a rede "Cristãos para o Socialismo".

Palavras-chave: socialcristianismo; pensamento dos anos 1960; redes intelectuais; circulação de ideias; "Economia e Humanismo"; "Cristãos pelo Socialismo"; exportações eidéticas.

\begin{abstract}
When it comes to understanding the position of Chilean and Latin American intellectuals, in the context of the global circulation of ideas, research on social Christianity in the 1960s is interesting, because it can be set at the confluence of several movements. In the process of understanding the syntheses produced in the context of social Christianity of that time, we discover the process leading to the combination of different lines of thought, taking into account the networks through which they appeared and combined. On the other hand, rather than conforming to the accepted idea of studying culture in our part of the world, as only the importers, we intend to study the exportation of these syntheses and the networks through which they traveled. This work focuses primarily on two cases: for the importation of ideas, the network "Economy and Humanism" and for the exportation, the network "Christians for Socialism."
\end{abstract}

Key-words: Social Christianity; thought of the 1960s; intellectual networks; circulation of ideas; "Economics and Humanism"; "Christians for Socialism"; eidetic exportations. 


\section{Introducción}

El estudio de la circulación de las ideas, a través de las redes intelectuales o de otros canales, ha ofrecido una renovada perspectiva para el estudio del pensamiento, particularmente en las regiones periféricas. A menudo, el estudio de las ideas en estas regiones fue abordado casi únicamente a partir de la noción "influencia".

La noción "hibridación" ha mejorado la de influencia, asumiendo la idea de una herencia entre un autor y otro, permitiendo formular mejor la producción de pensamiento y cultura, en las regiones periféricas.

La noción "redes intelectuales" nos permite entender un canal privilegiado a través del cual circulan las ideas, a la vez que el medio ambiente en el cual opera la intelectualidad ${ }^{1}$.

El pensamiento socialcristiano, como aquel pensamiento de cristianos que, asumiendo su condición de tales, se ocupan explícitamente de problemas económico-sociales, tuvo en Chile la mejor expresión política de América latina y más allá, dentro del mundo periférico en general. Otros países donde el socialcristianismo alcanzó formulaciones políticas exitosas han sido Costa Rica y Venezuela; menos significativas en Senegal y Tanzania. La expresión del social cristianismo político en Chile, cuyo mayor teórico fue Eduardo Frei M., no significó que en todas las ramas se dieran figuras de igual envergadura. Por ejemplo, una pedagogía socialcristiana tuvo en Paulo Freire, un brasileño, su mayor representante; una teología en Gustavo Gutierrez o Leonardo Boff, peruano y brasileño respectivamente; una filosofía en Enrique Dussel, argentino. En pensamiento social, sin embargo, probablemente el mayor teórico fue el belga, residente en Chile, Roger Vekemans, refiriéndonos siempre a América latina. Vekemans forma parte de la pléyade de extranjeros que, durante los largos 60s junto a $\mathrm{P}$. Freire, R. Prebisch, A. G. Frank, F. H. Cardoso, E. Iglesias, poco antes C. Furtado, contribuyeron a hacer de Chile una potencia intelectual de rango medio a nivel mundial, haciendo un aporte fundamental al boom de las exportaciones eidéticas chilenas.

1 Otras escuelas del pensamiento como el neoliberalismo, a pesar de tener figuras de alto reconocimiento internacional como Hernando de Soto, por ejemplo, no han logrado los niveles de reconocimiento de las anteriores. 
Este trabajo apunta a entender cómo fue posible que Chile se transformara en una potencia de rango medio en las exportaciones eidéticas. Para ello se utiliza el caso del pensamiento social cristiano, sin pretender ahondar en esta escuela más de lo imprescindible.

La noción "exportaciones eidéticas" puede entenderse al menos en dos sentidos: exportación de productos que vehiculizan ideas, como son libros y revistas; exportación de ideas que son efectivamente incorporadas en el pensamiento de personas de otras regiones. Es decir, la exportación de ideas puede medirse a partir de los criterios de la industria editorial, pero por otra parte puede medirse a partir de las citaciones en las regiones receptoras, de los autores y conceptos exportados. Ambos procesos se encuentran parcialmente relacionados, pero distan de ser idénticos: ha habido exportaciones de libros que nadie leyó y hubo recepción de ideas de unas regiones en otras por la vía de la oralidad. Para este efecto, es más interesante la exportación de ideas propiamente tal.

La noción "potencia intelectual de rango medio" caracteriza a un estado nación, aunque también podrían ser instituciones, que teniendo clara presencia regional (continente, países vecinos) y en otras partes del mundo, no alcanzan presencia global. En esta época las grandes potencias fueron USA, Francia, la URSS y quizás Alemania y Gran Bretaña. De rango medio India, China, Chile, quizás Cuba², Egipto, Brasil, y El Caribe no hispanófilo.

Entre los exportadores de rango medio existen algunos que son casi monoexportadores y monoproductores. Es el caso de China que casi exporta únicamente maoísmo. Chile posee una exportación relativamente diversificada: ciencias económico-sociales, pensamiento político, teorías pedagógicas, ideas teológicas. Por cierto, las grandes potencias exportan una gran variedad de disciplinas, paradigmas, escuelas y autores. Chile ocupó el vacío que se produjo por la baja de exportaciones de otros agentes y por su incapacidad para renovarse produciendo con nuevas tecnologías ${ }^{3}$.

2 Durante los largos 60s Cuba fue el otro país de América Latina que gozó de un boom de exportaciones eidéticas. Aunque fue casi mono exportadora de marxismo cubanizado (el pensamiento de F. Castro y E. Guevara), el interés por Cuba llevó a comprarle su ensayo literario-cultural (R. Fernandez Retamar, A. Carpentier, J. Lezama Lima, J. A. Portuondo y varios otros). Cuba no produjo ni ha producido casi otras disciplinas dentro de las ciencias sociales y humanas de categoría continental en el Siglo XX. No hubo importantes filósofos, sociólogos, economistas, politólogos ni teólogos.

3 Los mayores productores de ideas en Latinoamérica, Argentina, Brasil y México, no tuvieron buenos años 60 s en la exportación; aunque en verdad Brasil fue antes mucho más un productor 


\section{Redes intelectuales y circulación de las ideas en el espacio socialcristiano}

A diferencia de otros espacios, como el socialista, el de las ciencias económico-sociales, el de los estudios culturales, el espacio socialcristiano posee algunas especificidades que deben ser entendidas para explicar cómo circulan allí las ideas. La existencia de la Iglesia Católica, de otras iglesias, y las órdenes religiosas son fundamentales. Este aparato institucional es, a mediados del siglo XX, mayor que el de las universidades, partidos políticos u organismos internacionales, en el caso latinoamericano. Más claro es esto cuando iglesias y órdenes poseen también universidades y están asociadas, de algún modo a partidos políticos. Ello hace que la fluidez de la circulación de las ideas sea particularmente rápida, especialmente si se tiene en cuenta el alto nivel de profesionalismo y de recursos de la orden jesuita, la mayor potencia intelectual cristiana de América latina, de muy lejos, y para esos años comparable a la red cepalino dependentista, con la cual tiene algunos puntos de contacto y superior a todas las demás redes, por cierto a las de la III Internacional y a la masonería, por citar algunas de las importantes.

Algunas redes que contribuyeron a la entrada de discursos también contribuyeron a la salida, otras fueron específicas. Por ejemplo, la red Lebret-Economía-Humanismo trajo ideas a Chile pero llevó poco hacia fuera, la red Democracia Cristiana Latinoamericana y la jesuita importaron y exportaron; la red de educación liberadora más bien exportó, pues se trata de una red tardía. En Venezuela, en Bolivia, en Perú, en Costa Rica, en Nicaragua, por ejemplo, se recibe la síntesis generada en Chile, no sólo porque allí se lea Mensaje, Política y Espíritu, Teología y Vida u otras publicaciones producidas en Chile, sino también porque importantes teóricos chilenos o residentes en Chile exponen su pensamiento en esos países.

que un exportador, el problema del idioma se lo impidió sistemáticamente. Durante esos años, Argentina y Brasil fueron inhibidos por las dictaduras, y ello benefició Chile, y México no logró ponerse suficientemente rápido en la onda de las ciencias económico-sociales. Estas serían clave de la renovación del pensamiento por todo el Tercer Mundo en los 1960s, como también en el ámbito de quienes desde el primero se ocupaban del tercero. En esta renovación, que provino principalmente de USA, Francia y Gran Bretaña, y en gran parte de la intelectualidad judía residente en esos países (teorías del desarrollo, filosofía, neomarxismo, etc.), quedaron fuera los países socialistas, donde hubo pocas personas capaces de asumir las nuevas perspectivas. 


\section{El boom de las exportaciones eidéticas ${ }^{4}$}

El pensamiento cepalino-dependentista y el liberacionista son las dos escuelas que han puesto a América Latina en el mapa mundial de las ideas, en el ámbito de las ciencias sociales y las humanidades. Estas dos escuelas de pensamiento tuvieron en Chile un importantísimo espacio para su elaboración, especialmente la primera.

La exportación del pensamiento de R. Prebisch, C. Furtado, F. H. Cardoso, E. Faletto, A. G. Frank y O. Sunkel, entre muchos otros, impactó no únicamente por toda América Latina y El Caribe, sino que también en centros de estudios sobre el desarrollo en el Primer Mundo (véase Devés, 2004) y por cierto en varios países de África (véase Devés, 2005, 2006) y en otros tantos de Asia ${ }^{5}$. La exportación del pensamiento cristiano-social en la forma más moderada (Manuel Larraín y Roger Vekemans) y en la más radical (Cristianos por el Socialismo) tuvo amplia repercusión en América Latina y en varios lugares de Europa. El liberacionismo de P. Freire, ensamblado en Chile, gozó de inmediato impacto mundial ${ }^{6}$.

Por cierto, existen métodos cuantitativos y cualitativos para dimensionar el impacto de los autores y las escuelas de pensamiento. Realizar una investigación para medir exhaustivamente el boom de las exportaciones eidéticas chilenas en los largos 60s sería un trabajo del máximo interés. Este artículo irá aportando apenas algunos datos e indicadores que, utilizándose comparativamente (los largos 1960s versus otras décadas; Chile versus otros países), permitirán formular el problema que interesa.

4 La disciplina que estudia las ideas (eidología) aunque posee categorización propia, alude a conceptualizaciones provenientes de especialidades como la biología, la ecología, la economía o la estrategia entre otras, para poner en relieve dimensiones que con las propias categorías no se resaltan suficientemente. Una perspectiva conservadora quisiera trabajar únicamente con conceptos tradicionales: "influencias", "generaciones", "escuelas". Éstos, aunque válidos para entender algunos fenómenos, se hacen insuficientes para expresar otros o para preguntar o responder sobre cuestiones no convencionales.

5 En países como Kenia, Nigeria, Senegal y Tanzania pero también otros de África como Uganda y Ghana se recibió y reelaboró el pensamiento cepalino-dependentista, como un insumo para la creación del dependentismo africano. En el Asia autores de India, Pakistán, Bangladesh y Sri Lanka acusan también la presencia de la obra de los latinoamericanos en sus escritos.

6 El pensamiento de Paulo Freire debió, para alcanzar todas las proyecciones, exportarse desde una plaza mayor como fue Ginebra, desde el Consejo Mundial de Iglesias. Su obra, que ya se había exportado profusamente desde Chile, pudo exportarse masivamente al Tercer Mundo. Esta obra impactó tanto en figuras de primer nivel como el presidente de Tanzania Julius Nyerere (véase Devés, 2005) y miles de educadores populares anónimos. 


\section{OBJETIVOS}

1. Caracterizar (definir) los paradigmas socialcristianos elaborados durante los largos 60s, en contraste con los otros paradigmas socialcristianos que operaron en Chile durante el siglo XX.

2. Determinar desde dónde y a través de qué redes llegaron los componentes de esos paradigmas.

3. Determinar cómo (hacia dónde y a través de qué redes) fueron exportados esos paradigmas.

4. Entender las razones por las cuales una comunidad intelectual, que opera como "fabrica de ideologías" y como exportadora de productos con valor agregado, tuvo tal aceptación en el extranjero.

En definitivo, es el $4 .^{\circ}$ objetivo el que, en este trabajo, da sentido a los demás, porque el caso del cristianismo-social es una manera de estudiar la recepción, procesamiento y exportación de ideas.

\section{HIPÓTESIS}

1. Hubo dos paradigmas a) uno formulado a comienzos del período, que denominaremos "del desarrollo" y b) otro a finales del período, "de la liberación", que son suficientemente diferenciados aunque comparten algunos elementos, a la vez que el " $b$ " se construye sobre la herencia y la supuesta superación del " $a$ ".

2. Para el "a" los componentes económicos llegaron principalmente a través de la red "Economía y Humanismo" liderada por J. Lebret y de la red cepalina; los componentes filosóficos y políticos a través de la red demócrata cristiana que circulaba las ideas de J. Maritain y L. Sturzo; los componentes sociales: a través de redes latinoamericanas.

3. La exportación del segundo paradigma se dio principalmente hacia otros países de América latina, a través de la red liberacionista, traslapada en buena medida con la orden jesuita, pero también a través de algunos exiliados, particularmente en Costa Rica, Francia y luego Nicaragua.

4. El éxito de las exportaciones eidéticas socialcristianas, y chilenas más en general durante los largos 60s, debe entenderse sobre la base de cuatro factores que se articulan: las amplias redes existentes, el prestigio de la "marca Chile", la sensibilidad sesentista y la calidad del producto. 


\section{Los seis paradigmas socialcristianos en Chile durante el siglo $X X$}

El pensamiento socialcristiano que se ha manejado en Chile puede dividirse en los siguientes paradigmas (abusando algo del concepto) que se inician en fechas más o menos claras aunque se prolongan y coexisten parcialmente hasta fechas bastante indeterminadas.

El primero se articula desde los primeros años del siglo XX siendo su base teórica indiscutida la encíclica Rerum Novarum. Allí se intentan dos tareas: mejorar las condiciones de vida de los sectores populares urbanos y organizar a los trabajadores en forma alternativa a las socialistas y anarquistas, cristianizando a los obreros. El ideólogo de este movimiento es Guillermo Viviani y poco después Fernando Vives Solar.

El segundo se articula hacia 1930. A Rerum Novarum se agrega Quadragésimo Anno en 1931. Incorpora un componente corporativista y anti capitalista-liberal, insistiendo en la recuperación de las corporaciones medievales. Aparece la noción de deberes sociales de los católicos y se insiste en el sindicalismo cristiano. En esta línea se ubican Viviani y Vives pero también Eduardo Frei, Manuel Garretón, Ignacio Palma, Alejandro Silva Bascuñan y en general la Falange Nacional y la revista Lircay. El teórico Latinoamericano más reconocido en este paradigma es el brasileño Alceu Amoroso Lima (Tristão de Athayde).

El tercer paradigma se formula hacia 1950 suplantando la noción de corporativismo por cooperativismo y comunitarismo. Existe un reconocimiento de la democracia política con mucho mayor énfasis que en el anterior. Aparece la noción desarrollo y promoción popular y del campesinado, así como la de cambio estructural. Pierde fuerza o desaparece la noción de sindicalismo cristiano, la idealización de la edad media. Los ideólogos son Eduardo Frei, Manuel Larraín, Julio Silva Solar, Jacques Chonchol y, algo tardío, Roger Vekemans. A nivel latinoamericano existen varias figuras: Lino Rodríguez Arias, Rafael Caldera y con menos fuerza que antes, Alceu Amoroso Lima. Una expresión importante es Chile voluntad de ser, carta pastoral de los obispos en 1962.

El cuarto paradigma se formula hacia 1970, a partir de nociones como dependencia, liberación, socialismo y concientización. Sospecha o descree de la democracia política. Postula la revolución como método para 
el cambio estructural, luchas de liberación. Los ideólogos son Gonzalo Arroyo, Pablo Richard, con menos fuerza Julio Silva Solar y Jacques Chonchol; Paulo Freire y Hugo Assmann, brasileños, pero residentes en Chile durante varios años; Franz Hinkelammert, alemán, pero también residente en Chile. Las figuras latinoamericanas más importantes Helder Câmara, Gustavo Gutiérrez y Juan Luis Segundo.

El quinto se formula durante la segunda mitad de los 1970s, hereda más del tercero que del cuarto, aunque recibe de ambos. Su noción clave es derechos humanos, cuestión que articula con democracia, apunta a defender la vida en general y los derechos civiles, políticos y económico-sociales. Sus ideólogos son Jaime Castillo, Raúl Silva Henríquez, José Aldunate ${ }^{7}$. A nivel latinoamericano debe mencionarse a Adolfo Pérez Esquivel e Ignacio Ellacuría, español residente en El Salvador.

El sexto se formula hacia 1990, poco antes apuntando hacia la cuestión de la multiculturalidad y el derecho a la diferencia, particularmente de los sectores populares e indígenas. Destaca los movimientos sociales, una dimensión ecológica o medio-ambiental ligada al tema de la vida, más que de la noción de derechos como en el quinto. En Chile, algunos ideólogos son Diego Irarrázabal y Ronaldo Muñoz. A nivel latinoamericano, Leonardo Boff y Enrique Dussel.

\section{2. ¿Cómo se importan las ideas? Las redes de entrada}

Para discutir este asunto se comenzará por desmontar el tercer paradigma social cristiano, ese que madura entre 1955-1965, aunque se extiende por un tiempo mayor. Los componentes son: 1. desarrollo, 2. cambio estructural, 3. comunitarismo, 4. democracia política, 5 . neotomismo, 6. reforma agraria, y poco más tarde, 7. integración latinoamericana, 8. promoción popular.

Sería abusivo sostener que todos los componentes del paradigma 3 fueron importados. De hecho, varios de ellos se encontraban presentes en Chile antes de 1950, como por ejemplo el neotomismo. No es menos cierto

7 Para más información sobre la expresión de este paradigma véase Aldunate (1944 y 2000) y Castillo (1986). 
que algunos de éstos, como el propio neotomismo, habían sido importados durante los 1930s y 1940s. Otros, como el integracionismo, habían sido reelaborados a fines de los 1950s y comienzos de los 1960s, a partir de nuevas hibridaciones, e incluso una versión anterior, como la aprista, había sido importada en los 1930s y 1940s, a través de redes distintas de las que habían traído el neotomismo. Es decir, se trata de descubrir a que redes internacionales se ligaron los socialcristianos para obtener esos insumos eidéticos con que compondrían el paradigma 3 lo que averiguaremos por partes (véase Sol Serrano, 1984).

Algunos de estos elementos, el 1 y 2 se encuentran presentes tanto en los constructos manejados por la red "Economía y Humanismo" como por la red cepalina. El número 3 proviene de "Economía y Humanismo" de modo único. El 4 y el 5 de la Democracia Cristiana europea, particularmente de las obras de Jacques Maritain y Luigi (Dom) Sturzo. El 6 y el 7 provienen más inmediatamente del cepalismo que retomó en buena medida estas propuestas de la herencia del pensamiento aprista clásico, que había tenido en Gabriela Mistral una de sus ideólogas. El 8 es un aporte Roger Vekemans. Pero debe ahondarse un poco más.

1. La noción desarrollo, como desarrollo económico, es fundamental en la asunción de un cambio de paradigma donde la justicia pasa por el crecimiento en primer lugar y por la repartición de lo existente. Esto supone la adopción de una perspectiva ética. Es decir, se produce una secularización del discurso: un papa puede decir que debe haber justicia pero no puede decir como generar desarrollo, aunque pueda afirmar condiciones, como por ejemplo respecto al reparto de sus frutos. Sea como fuere esto abre una dimensión técnica que antes no consideraba el socialcristianismo. Este cambio se ha dado en el pensamiento social cristiano francés y lo encarna Joseph Lebret, quien a adoptado la noción a partir de la escuela económica del desarrollo, cosa similar a la que ha ocurrido con Prebisch, que transitó desde la economía clásica al keynesianismo y desde allí al desarrollismo, realizando a éste aporte original. Las ideas de Lebret y Prebisch no son idénticas pero ambas manejan como un concepto clave la noción de desarrollo.

2. La noción cambio estructural también se encuentra en Lebret y en la CEPAL y proviene de la tradición socialista, de la que es heredera en cierta medida, el pensamiento desarrollista, creado por británicos y franceses principalmente, pensando en la realidad de sus colonias y no en 
Latinoamérica. Esta idea pretende que para mejorar los niveles tanto de producción como de justicia se requieren cambios en la estructura social: propiedad de los bienes, administración de éstos, relaciones entre las clases, movilidad social.

3. El comunitarismo es privativo y original de Economía y Humanismo. Viene a retomar o asociarse a corporativismo y cooperativismo haciendo a ambos conceptos más cristianos y más amables. Comunitarismo se liga a una cuestión de búsqueda de primitivismo cristiano y afectividad que no se encontraba en la noción de corporativismo, ya obsoleta por su asociación con el fascismo, ni en la de cooperativismo, demasiado laica.

4. La democracia se encontraba sin duda presente en la trayectoria política nacional y no era necesario ir a buscarla fuera. Sin embargo, en el modelo social cristiano de los años 30 no existe sino como resabio liberal. Para que sea recuperada en el ideario socialcristiano de fines de los 1950s debe provenir de fuentes cristianas y europeas y ello es lo que permite la obra de Maritain y de la Democracia Cristiana italiana. En esas fuentes se halla la revalorización de la democracia como sistema político, como negación del fascismo y de los sistemas comunistas. En todo caso, ya en La Política y el Espíritu de Eduardo Frei, en 1940, aunque con múltiples precauciones, existe una valorización de la democracia.

5. El neotomismo se recibe de las mismas fuentes que la democracia pero con cierta anterioridad. Ya en los 30 s y los 40 s se habían comenzado a recibir en Chile las ideas de Jacques Maritain, reformulando en tomismo con el fin de responder a desafíos de la sociedad del siglo XX. La noción de "humanismo integral" había hecho su efecto en Francia, en Chile y en otros países.

6. La reforma agraria, en la forma establecida en la ley es tardía, de mediados de los 60s, pero en formulaciones menos precisas, la necesidad de modificar el sistema agrario se encuentra en el pensamiento socialcristiano chileno con bastante anticipación. Esto tiene que ver con la distribución de la tierra, con el sindicalismo, con el establecimiento de formulas comunitarias y/o cooperativas. Son relevantes en este sentido las proclamas respecto a los sucesos de Lontué en 1951 y las declaraciones (y acciones) de Manuel Larraín. Es de notar que los obispados de varios países latinoamericanos se ocuparon del tema agrario y que la revista Mensaje entre fines de los 50s y comienzos de los 60 s reproduce varias comunicaciones. Debe notarse 
también la realización del $4 .^{\circ}$ Congreso Internacional Católico de la Vida Rural en Santiago en $1957^{8}$.

Por cierto, estas ideas provienen de fuentes muy variadas, pero una privilegiada debe ser la obra de Gabriela Mistral, importante para los jóvenes falangistas y para Frei en particular (FREI, 1989). Gabriela fue heredera y promotora de un pensamiento agrarista, que se difundió en Latinoamérica luego de la Revolución Mexicana, a través de las redes articuladas al movimiento aprista, aunque este pensamiento se hallaba en pensadores ácratos, socialistas, y comunistas no ligados al aprismo (véase en Devés, 2000, p. 117ss y 163ss).

7. El integracionismo, tardío en el socialcristianismo chileno, proviene de orígenes y redes similares a los del agrarismo. Dicho en términos más amplios, la vocación latinoamericanista en el socialcristianismo, reemplazando parcialmente el "occidentalismo conservador", es tardía.

En Chile el socialismo había asumido una perspectiva latinoamericanista desde comienzos de los 1930s. Es significativo que existió por esos años un importante exilio aprista en Chile, conectado a parte importante de la intelectualidad. En la declaración de principios de la Alianza Popular Revolucionaria Americana, se hallaba la reivindicación de la unión continental. Gabriela Mistral participó y difundió este ideario.

8. La promoción popular, como oposición a la marginalidad, es también una idea tardía. Es al parecer la más tardía de todas y debe esperarse a Roger Vekemans para que aparezca con toda la fuerza, a mediados de los 60s. Tanto es así que puede señalarse que se ubica en el tránsito del paradigma 3 al 4, en la medida que se hace presente el discurso sociológico sesentista.

Para justificar la importación de ideas se profundizará sólo en la red "Economía y humanismo", a través de la cual circularon las primeras tres nociones de este paradigma: desarrollo, reforma estructural y comunitarismo. Entonces ¿cómo funcionó la red "Economía y Humanismo" para circular algunas de sus ideas hacia Chile y cómo funcionaron los social

8 A quien interese detenerse en cómo se constituye este tercer paradigma sugiero leer el trabajo de Jacques Chonchol (1958) presentado precisamente en el $4 .{ }^{\circ}$ Congreso Internacional Católico de la Vida Rural. Allí se advierte como la doctrina social de la iglesia se va expresando a través del lenguaje de las nuevas ciencias económico-sociales. A la trayectoria comunitarista Chonchol va a agregar la cepalina, aludiendo explícitamente a la noción de "deterioro en los términos del intercambio" y aplicando la noción de "desarrollo" al tema agrario. 
cristianos de los años $50 \mathrm{~s}$ y primeros $60 \mathrm{~s}$ para articularse a ésta (y otras) redes internacionales?

Para entender este fenómeno es necesario comenzar destacando la figura de Joseph Lebret (1897-1966), inspirador y motor móvil de "Economía y Humanismo". Esta asociación se fundó en Francia (Marsella) en 1941 para estudiar las realidades económicas y sociales, motivar trabajos científicos capaces de elaborar una doctrina espiritualista, poniendo la economía al servicio del hombre y suscitar técnicos o profesionales capaces de determinar las condiciones concretas del bien común. Los medios para estos objetivos eran: la creación en Francia y en el extranjero de centros de estudio; la creación de escuelas de formación para la acción, publicaciones de revistas, boletines, documentos y colecciones; organización de cursos y otros medios de propaganda, enseñanza y acción (MALLEY, 1969, p. 50-51).

En 1942 aparece el manifiesto del grupo reunido en torno a Economía y Humanismo en el cual se alude a la ruptura de las comunidades naturales y del orden tradicional, al caos económico, a la desnaturalización del ser humano por la economía de la ganancia, a la idea de la sociedad moderna como un organismo enfermo. Luego de haber rechazado la opción liberal y la social estatal se proponía la comunitaria (MALLEY, 1969, p. 53-54). En mayo de 1942 apareció la revista Economía y Humanismo. En 1947, entre mayo y septiembre Lebret impartió un curso en la Escuela Libre de Ciencias Políticas de Sao Paulo, así como visitó a otros países de Sudamérica. En 1949 aparecieron otras dos publicaciones periódicas El diagnóstico económico y social e Ideas y fuerzas; en 1960 aparecería Desarrollo y civilización. Junto a estas publicaciones periódicas se pone en funcionamiento la casa Ediciones Obreras y el servicio Intercambio América Latina tendiente a financiar desde Francia la acción de militantes de la Acción Católica. Este servicio inició tempranamente algunos planes en Brasil ${ }^{9}$.

Lebret estuvo en Chile en tres oportunidades, en 1951 en un viaje de investigación, luego en 1965 con motivo de una reunión en Viña del Mar de la FAO y en 1966 para participar en la Semana Social. Entre 1947

9 Sobre este servicio argumenta Michel Quoist que se ha creado porque "es necesario ayudar a América Latina a salvarse ella misma, entre otras cosas permitiéndole dedicar como permanentes para la Acción Católica y la catequesis algunos de sus mejores militantes. Este es el objetivo que busca el servicio" (s/f, p. 188). 
y 1966 estuvo varias veces en Brasil ${ }^{10}$, y una vez, al parecer, en Argentina, Colombia, Uruguay y Venezuela (MALLEY, 1969, p. 254ss).

Sintetizando estos datos tenemos los siguientes elementos: existencia de un organismo central en Francia, varias publicaciones periódicas y otra documentación, reiterados viajes de investigación, cursos, participación en congresos y encuentros. En un texto de 1956, Lebret afirma haber estado en 60 países. Esto permite hacerse la idea de las redes en que participaba ${ }^{11}$.

La primera vez que se ha detectado la mención de Lebret en Chile es por parte de J. Silva Solar y J. Chonchol en un texto de 1948, Qué es el social cristianismo ${ }^{12}$.

\section{3. ¿Cómo y hacia donde se exportaron estas ideas? Las redes de salida}

En el acápite anterior se ha trabajado sobre el tercer paradigma socialcristiano operante en Chile, viéndose los procesos de importación de algunos de sus componentes. En éste se aborda el cuarto paradigma, intentando determinar cómo se exportó y hacia donde. No se desprenda de ello que el tercero sólo importó y el cuarto únicamente exportó. Se trata apenas de un procedimiento para mostrar ambas dimensiones, en la

10 En Brasil, Lebret con un equipo de sociólogos y la Fundación León XIII, trabajó en las favelas de Río de Janeiro. Uno entre quienes se impactaron con las ideas y la práctica del francés y pretendieron seguirlo fue el obispo auxiliar de Río en ese momento, Helder Câmara (SCHILLING, 1972, p. 11).

11 León J. Moreau, dominico como Lebret y seguidor de éste, en un artículo reproducido en Política y Espíritu en 1952, destaca algunos aspectos internacionales de la obra de su mentor. Lebret entre 1929 y 1939 realizó encuentros sobre los trabajadores marítimos en Francia, Bélgica, Holanda, Alemania, Dinamarca, Noruega, Inglaterra, Irlanda, Argelia, Tunes e Italia. Mas adelante señala que los equipos de trabajo se desarrollaron principalmente en Francia, pero que el movimiento se ha extendido por Bélgica, Suiza, el norte de África, Brasil, Colombia, Uruguay, etc. Concluye que "si tomamos en cuenta la importancia que tienen y tendrían aún más en el porvenir, Sud América en general y particularmente Chile, en la evolución histórica de la humanidad, no podremos tener duda ninguna tocante a la oportunidad o mejor dicho a la necesidad del establecimiento y del desarrollo de Economía y Humanismo en este país" (1951, p. 249 y 253$)$.

12 "Dos son las fuentes históricas principales de donde emana la tendencia socialcristiana de nuestra época: las enseñanzazas de León XIII, Pio XI y Pio XII y el pensamiento y la acción pléyade de ilustres católicos entre los cuales podemos citar a Ozanam, Lacordaire, De Mun, La Tour du Pin, Gibbons, Manning, Peguy, Chesterton, Candijn, Sturzo, Berdiaeff, Maritain, P. H. Simon, Tristan de Ataide, el Cardenal Suhard, Lebret, Barbu, etc." (SILVA; CHONCHOL, 1948, p. 24). 
medida que lo que pretende el artículo no es detenerse en cada aspecto del socialcristianismo sino principalmente ir a la cuestión de la circulación de las ideas a través de redes intelectuales.

Los elementos que componen el cuarto paradigma son en síntesis: 1. Liberación, 2. Socialismo, 3. Revolución o cambio estructural, 4. Dependentismo, 5. Marxismo estructuralista (Althusser) y humanista (Fromm), 6. Justicia social, 7. Antiimperialismo, 8. "Primitivismo" cristiano.

Estos son los componentes de lo que se llamó el cristianismo de izquierda hacia 1970 y por cierto ello se exportó como conjunto, pero esto debe formularse más específicamente, pues no podría decirse que fueron los cristianos de izquierda chilena los que exportaron el antiimperialismo, no son exportadores relevantes de este componente, aunque haya ido incluido en su propuesta. Lo más específico es un liberacionismo-dependentista-socialista, que aunque se estaba produciendo también en otras partes, en el medio chileno se produjo de manera más nítida. Tampoco fue lo único que se exportó en este rubro, pues si se considera la obra de P. Freire producida en el medio chileno, especialmente La educación como práctica de la libertad (1967), una obra de inmenso impacto en Chile y fuera, ésta no corresponde estrictamente a dicho paradigma. En la obra del brasileño el componente dependentista tiene bajísima presencia y el socialista poca, a pesar de ser una obra clásica del liberacionismo, aunque temprana para el efecto que interesa. Es decir, Chile exportó diversos productos de corte liberacionista, unos con menos valor agregado a otros con mayor. Por ejemplo, el producto freiriano contiene componentes que Freire traía desde Brasil, inspirado en lo que estaba haciendo el Instituto Superior de Estudios Brasileños (ISEB), que ensambló en Chile agregándole poco más ${ }^{13}$. El producto exportado por

13 El freirismo fue un boom espectacular a cuatro años de su publicación, por ejemplo en Francia, La educación como práctica de la libertad se había editado ya 13 veces. Pasa con Freire algo similar a lo que ocurre con Gustavo Gutiérrez y su Teología de la liberación, obra maciza que es captada inmediatamente por el mercado intelectual internacional, editada y comentada por todas partes, y a lo que ocurre con Dependencia y desarrollo de Cardoso y Faletto. Debe destacarse la incapacidad (o desinterés) de Freire por incorporar tanto en La educación como práctica de la libertad (redactada antes parcialmente, pero reelaborada en Chile entre 1965 y 1966) y en Pedagogía del oprimido (redactada en Chile entre 1967 y 1969, con algunos agregados posteriores) la obra de personas con las cuales dialogaba constantemente: F. H. Cardoso, J. Chonchol, A. Affonso e incluso F. Weffort, que es el único que cita en esas obras (1987, p. 93). Menciona también en Aprendiendo con la propia historia (1987) sus contactos con Ernani Fiori y con los hijos de éste Jorge y José Luis, todos residentes en Chile, aunque prácticamente no alude a la obra de ellos. Cita eso sí a autores que eran leídos en Chile en los años que estuvo en el país (fines 1964-fines 
Cristianos por el Socialismo es elaborado en Chile, obviamente teniendo en cuenta no sólo elementos fabricados en el país, sino que algunos importados y otros hechos en Chile, pero el diseño general es de factura nacional.

Como es sabido, en numerosos lugares de América latina se estaban generando movimientos similares al de Cristianos por el Socialismo, aunque no tuvieran las dimensiones ni la caja de resonancia de Chile ${ }^{14}$. Entre el 23 y el 30 de abril de 1972 se realizó en Santiago el Primer Encuentro Latinoamericano de Cristianos por el Socialismo, en el cual se ha dicho que participaron más de 400 personas de todos los países de América latina (DOCUMENTO FINAL, 1972, p. 205).

En el documento producido cristaliza el paradigma del cual se encuentran obvios antecedentes en la declaración a la prensa de abril de 1971 del grupo de los 80 y en las de la Iglesia Joven, por ejemplo, en su declaración de octubre de 1970. Pero no solamente son similitudes ideológicas sino que hay una clara factura chilena en el documento, que se percibe al

1969) como por ejemplo F. Castro, E. Guevara, Mao, L. Goldman, L. Althusser, E. Fromm, H. Marcuse, cosa que se advierte tanto por las fechas de las ediciones referidas, como por el listado de las obras de su biblioteca destacadas en las notas de Ana María Araujo Freire en las Cartas a Cristina (1994) de Freire.

Freire está pensando Brasil, y por extensión América latina, a partir de trabajos publicados entre 1915 y 1945, que eran los insumos históricos y sociológicos del ISEB, F. Oliveira Viana que publicó antes de 1920, Gilberto Freyre a mediados de los 1930s, Fernando de Azevedo, hacia 1940. Es cierto que cita igualmente a Florestan Fernándes que publicó en los $1950 \mathrm{~s}$ y $60 \mathrm{~s}$.

Es muy relevante, que a diferencia del medio intelectual latinoamericano de los 60 s, Freire cita Los condenados de la tierra de Frantz Fanon (caribeño francés que residió y escribió en Francia, Argelia y Tunes) y Colonizador y colonizado de Albert Memmi (tunecino).

En otras palabras, para Freire su vida en Chile fue muy importante para agitar su pensamiento y ayudarle a formular sus ideas y para aportarle lecturas que no conocía antes, pero no existe ni influencia ni reelaboración de la obra de chilenos ni de latinoamericanos residentes. No es menos importante la impermeabilidad a la producción de las ciencias económico-sociales que estaban marcando la época. Freire no cita ni a sus compatriotas C. Furtado, H. Jaguaribe, F. H. Cardoso ni a figuras como R. Prebisch, G. Germani, A. Pinto u O. Sunkel.

En cierta manera la producción de Freire en Chile viene a falsear mi propia tesis. Freire fue un gran exportador de ideas que se formularon en el ecosistema intelectual chileno, en el riñón de los largos 60s, pero la producción chilena o en Chile no le sirvió para hacer hibridaciones. Sólo utilizó autores leídos y citados en Chile, pero no chilenos ni residentes. Fue principalmente la sensibilidad que se vivía, como ecosistema intelectual, lo que le motivó, pero no la producción generada en Chile.

14 "Sacerdotes para el Tercer Mundo" en Argentina, ONIS en Perú, Golconda en Colombia, "Sacerdotes para el pueblo" en México. Se reconoce como antecedente en Chile el movimiento "Iglesia Joven". Ahora bien todos éstos tuvieron un carácter nacional, aunque se asumía que en otras partes de América latina existían grupos con carácter similar. Una innovación de Cristianos por el Socialismo fue constituirse en un movimiento de dimensiones latinoamericanas, agrupando y radicalizando estos movimientos. En tal sentido se va creando una red que favorece la circulación de las ideas siendo los chilenos, aunque no los más fuertes en propuestas teológicas, sí los más orgánicos. 
compararlo con los anteriores, de los cuales es una ampliación y maduración, a la vez que al compararlo con el de grupos de otras nacionalidades donde se abordan tópicos y se usan conceptos que en éste no aparecen. Señalado lo anterior, ¿a través de que canales se exporto este material?

La red latinoamericana de cristianos por el socialismo, construida en partes sobre contactos anteriores en parte recientes, fue el canal principal de difusión del pensamiento cristiano-socialista chileno. Porque ofreció un público amplio para estas ideas; porque permitió que esas personas llevaran hacia otros países las ideas y los documentos, porque haciéndose más fluida la comunicación pudieron desde muchos lugares continuar recibiendo las publicaciones chilenas; porque los ideólogos chilenos recibieron invitaciones y, luego de septiembre de 1973, fueron invitados o acogidos en exilios. Pero sin duda no se trató del único canal, Cristianos por el Socialismo se beneficiaron igualmente de las vastas redes tejidas desde y hacia Chile por intelectuales de diversas procedencias disciplinarias, ideológicas y geográficas. En una edición que se hizo en 1972, el mismo año del encuentro latinoamericano, de varios documentos, los editores incorporan la información que poseen sobre las ediciones del documento final que suman 20, aparte de la edición masiva en Chile que fue de 20 mil ejemplares. En el extranjero se editó en español, francés, inglés, italiano en países de América Latina, Europa y Asia ${ }^{15}$.

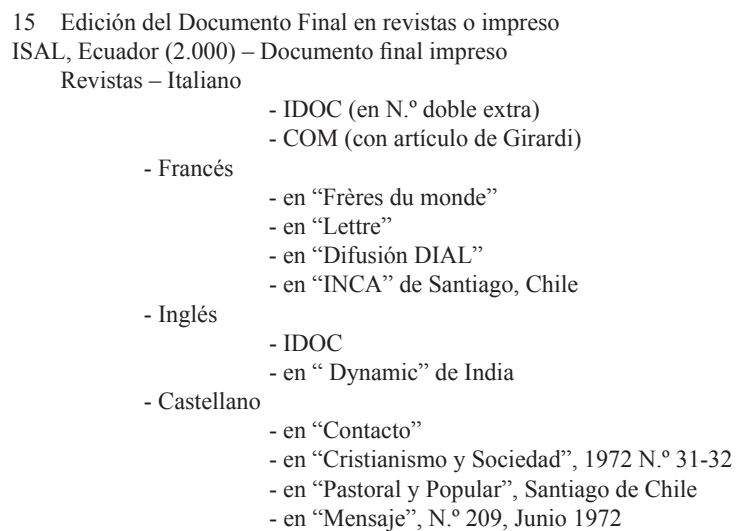




\section{4. ¿Por qué pudo exportarse el producto y cómo pudo producirse el boom de las exportaciones eidéticas chilenas?}

El problema de este trabajo ha sido mostrar y explicar parcialmente cómo opera la circulación de las ideas, importación y exportación, hacia/ desde un respectivo ecosistema. Para esto se ha ejemplificado a partir de los paradigmas socialcristianos operantes en los largos 60s en Chile.

1. La pregunta más relevante, en este sentido, es sobre la forma en que un pequeño país como Chile pudo transformarse, durante una década, en una potencia intelectual de rango medio, a nivel mundial.

El desmedido desarrollo de las fuerzas productivas intelectuales del Chile de esos años (parecido a esos booms efímeros que experimentan algunas regiones cuando se descubre oro o diamantes) debe ser entendido en el marco del sistema global de los $60 \mathrm{~s}$. La sensibilidad sesentista permitió que se concentraran fuerzas, así como hizo que esas fuerzas repercutieran sobre el mundo de modo diferente a como habría ocurrido sin tal sensibilidad ${ }^{16}$.

El calentamiento global de la sensibilidad durante esa época generó las mayores tormentas intelectuales del siglo, sólo comparables a las de los años de la primera post guerra mundial, pero superiores.

Ahora bien, de lo que se trata es de capitalizar una experiencia para repensar la actividad intelectual chilena actual y más allá. ¿Cómo imaginar, décadas después de este boom, las proyecciones del quehacer intelectual

- en "Marcha", Montevideo

- en "Hechos y Dichos", España N. ${ }^{\circ}$ 430, Junio 1972.

Dos textos - Secretariado de Cristianos por el Socialismo. - Pasos, ISAL.

Edición mimeografiada ed. En francés - IDOC Internacional, Roma

- Centre France Amérique Latine, Paris

ed. En Italiano - IDOC (AAVV 1972,9)

16 No se ha elaborado, hasta donde conozco, una teoría de los ciclos de la sensibilidad y las ideas en el proceso de mundialización. 1800, poco antes poco después, 1850,1920, 1965 son picos en los flujos del calentamiento de la sensibilidad; por cierto, son inseparables, de algunas revoluciones. Sin duda han estado marcados, especialmente durante el Siglo XX, por la irrupción del juvenilismo y las tormentas políticas. Obviamente, hay hechos económicos que se articulan, pero solo cuando se dan condiciones y en ningún caso parecen ser la causa. ¿Qué causa económica común podría tener el levantamiento de la juventud chilena o china, checoslovaca y usamericana, francesa y mexicana, sudafricana y española, a mediados de los 1960s? 
chileno? ¿Por qué en unas épocas nuestra producción intelectual ha sido tan demandada en el exterior, o ha tenido tal aceptación, y en otras no? O dicho de otro modo: a la hora de potenciar la colaboración intelectual en el Conosur y en toda Latinoamérica, puede o no inspirarnos la capacidad del medio intelectual sesentista para exportar su producción ${ }^{17}$. Para responder parcialmente acaso a estas preguntas vamos a las causas que permitieron las exportaciones del cuarto paradigma cristianismo social.

Esta pregunta del porqué fue vendido o exportado este paradigma debe entenderse en dos sentidos que son complementarios: por qué fue acogido, es decir, porqué influyó en gente que lo hizo fuera de Chile. Y por otra parte, porqué despertó interés, siendo recibidas las personas y citados los trabajos, aunque no se asuma como influencia. En otras palabras, la acogida de la idea tiene dos dimensiones que se potencian: hay quienes la reciben como un insumo para su propio pensamiento y hay quienes la reciben con interés (compran libros, invitan a expositores, la citan) aunque no pasa a formar parte viva de su pensamiento, sino que es objeto de interés académico o político.

1. La facilidad de las exportaciones se debió, en primer lugar, a la fluidez de la comunicación existente con el extranjero, la fluidez de la comunicación a través de redes como la del CELAM; de la orden jesuita; de los belgas en América latina y ex estudiantes en Bélgica, especialmente de la U. de Lovaina; Economía y Humanismo, entre otras, hicieron esto más fácil ${ }^{18}$. Cristianos por el Socialismo exportó sus ideas a partir de los contactos de estas redes y por cierto incorporó otros, a la vez que reestructuraba las redes de intelectuales cristianos.

17 La pregunta por las razones del boom de exportaciones y por las posibilidades para generar colaboración intelectual no debe hacer creer que la comunidad intelectual es tanto mejor cuanto más productos eidéticos exporta. Sin duda la exportación de sus productos es una medida de su valor, pero sólo una de las medidas. Cuestiones como la capacidad de entender a su sociedad y de contribuir a que ésta se autocomprenda y de mejorarla, en la medida que ésta asume las propuestas de esa intelectualidad, son más relevantes que la capacidad exportadora. En todo caso debería suponerse que la intelectualidad que genera buenos productos los exportará a otras sociedades.

18 Quien más activó e interconectó estas redes fue Roger Vekemans. Su capacidad intelectual, su dominio de varias lenguas, su capacidad para captar recursos y energía, le permitieron contactarse con enorme cantidad de gente, mover múltiples iniciativas, interconectar redes, etc. Estas redes por cierto eran heterogéneas e importaron y exportaron ideas con las cuales Vekemans no comulgaba. Sugiero leer desde esta perspectiva Vekemans 1982, entre una maraña de dimes y diretes aparecen las conexiones intelectuales del autor, relevantísimas para los largos 60s. 
2. El producto exportado fue elaborado en buena medida por extranjeros residentes. Para decirlo de otro modo, los extranjeros participantes en grupos como Iglesia Joven, Grupo de los 80, Cristianos por el Socialismo contribuyeron a elaborar un producto que estaba en sintonía con los que sectores de consumidores de idea, en sus países de origen, estaban demandando. Dicho todavía de otra manera: delegados de los compradores participaron en la elaboración del producto a exportar.

3. La marca Chile estaba, para ciertos segmentos, cargada de prestigio. Para el cristianismo progresista de América y de ciertos países de Europa, los productos eidéticos precedentes desde Chile eran altamente apreciados.

4. El producto "liberacionismo cristiano socialista" chileno era altamente demandable dada la sensibilidad que valorizaba tal genero de productos. Así logró insertarse en nichos no sólo latinoamericanos sino también en el Primer Mundo, en el seno de grupos que gestionaban formas de colaboración con el Tercer Mundo ${ }^{19}$. El pensamiento cristiano social chileno logró poco impacto comparativamente en las comunidades latinas y negras usamericanas donde la teología de la liberación más académica fue mejor recibida y por esa vía pasó a Sudáfrica para alguna información sobre la teología de la lideración y su impacto en Sudáfrica ${ }^{20}$ y probablemente a $\mathrm{Asia}^{21}$.

5. Se trató de un producto elaborado con profesionalismo y que encontró su público entre los animadores de comunidades de base, los clérigos dedicados a cuestionar pastorales, las agrupaciones políticas y las ONGs cristianas. Este era el público de G. Arroyo, P. Richard (Esteban Torres) D. Irarrazabal y R. Muñoz. El producto estaba fabricado profesionalmente y destinado a este público y no al mundo propiamente académico. Este último consumía de preferencia la producción del peruano G. Gutierrez, de los brasileños H. Assmann y más tarde L. Boff, de argentinos J. C. Scannone y más tarde E. Dussel y de los hispaldoreños I. Ellacuría y J. Sobrino.

19 En el caso del pastor protestante francés Casalis, citado por F. Moreno Valencia, quien además de explicar la posición del movimiento chileno proyectaba lo que debía ocurrir en Europa: "Es evidente que lo que se pide a los cristianos por el socialismo europeo es tener una praxis efectiva en la lucha socialista" (MORENO-VALENCIA, 1985, p. 60).

20 Para alguna información sobre la teología de la liberación y su impacto en Sudáfrica, véase por ejemplo Boesak, 1981.

21 Véase a este respecto Berryman (1989, p. 151) donde cita a teólogos de la liberación srilanqueses como Tissa Balasuriya y Aloysius Pieris. Este último se permite incluso cuestionar la perspectiva occidentalizante de J. P. Miranda y Jon Sobrino. 
6. El producto final de Cristianos por el Socialismo incorporó aportes del pensamiento dependentista y socialista, entre otros. Se trataba de piezas con marcas registradas, que traspasaban su prestigio al producto final. Es decir, el producto fabricado por Cristianos por el Socialismo no fue comprado únicamente por sus componentes cristianos, en países componentes específicos sino también por todas las piezas comprendidas. Incluso había no cristianos que lo compraban sólo por las piezas, desensamblándolo y usando lo que les interesaba ${ }^{22}$. Por lo demás esto ocurre con casi todos los productos eidéticos. Pocos son los que se compran para usarlos como totalidad. Para decirlo de otro modo: este producto incorporó las "nuevas tecnologías" en el pensamiento latinoamericano, que fueron las ciencias económico-sociales.

7. El hecho de presentarse y ser percibido como parte de una producción latinoamericana, relativamente autóctona, le ganó un interés asociado a la búsqueda de "autenticidad" de los propios latinoamericanos y a la búsqueda de "exotismo" del Primer Mundo. Por una u otra razón el producto se hacía novedoso e interesante, y ello se sumó a los otros prestigiadores.

8. No debe despreciarse el hecho que los propios Cristianos por el Socialismo se ocuparon de poner su producto en el exterior. Esto tuvo 5 expresiones, al menos: difundiendo la convocatoria al encuentro, lo que traería compradores para sus ideas; la realización del encuentro durante la reunión de la III UNCTAD, lo que aseguraría la publicidad, por la amplia cobertura periodística; el envío del material al exterior para que fuera reproducido; y la posterior organización de reuniones en que se presentaron las ideas ${ }^{23}$; los viajes y exilios de algunos miembros lo que permitió una difusión mas decantada ${ }^{24}$.

22 En el caso por ejemplo, de Leopoldo Zea. Este como pensador laico no se interesaba en el componente cristiano o teológico propiamente tal. "Compró" la teología de la liberación como parte de un movimiento de ideas emergido en América Latina en busca de desalienación (ZEA, 1976, p. 533).

23 Probablemente el mayor organizador de reuniones hacia fuera de América latina, especialmente hacia Estados Unidos y en conexión con teólogos africanos y asiáticos fue Sergio Torres. Muy importante por la convocatoria que tuvo fue la reunión realizada en Detroit, en agosto de 1975 (TORRES; AGLESTON, 1980).

24 El grupo más importante es el que se instala en Costa Rica, en el Departamento Ecuménico de Investigaciones (DEI) donde se instalan Pablo Richard y Franz Hinkelammert al cual se suman otras personas como Helio Gallardo. Pero también en Paris estuvieron durante algunos años Gonzalo Arroyo y Pablo Richard. 
Ahora bien las razones específicas que explican la exportación del liberacionismo chileno o la ideología de Cristianos por el Socialismo no bastan para explicar el boom de las exportaciones eidéticas chilenas durante los largos 60s. Estas razones deben por tanto ampliarse o extrapolarse y, por cierto, complementarse con otras razones que favorecieron la exportación de productos eidéticos diferentes y que contribuyen a una explicación del fenómeno como totalidad.

1. La marca Chile no operó sólo como portadora de prestigio para ambientes cristianos-progresistas sino mucho más ampliamente para ambientes de centro e izquierda de variado pelaje doctrinario. $Y$ esto es en doble sentido, Chile aportaba prestigio, pero la pléyade de intelectuales y políticos chilenos y extranjeros residentes beneficiándose de ello, contribuían también al crecimiento de tal prestigio. El prestigio de Chile no era anterior sino correlativo al prestigio de su capa político intelectual. Eduardo Frei M. y Salvador Allende exportaron pensamiento político, como Roger Vekemans y Gonzalo Arroyo exportaron pensamiento cristiano social, como R. Prebisch y Osvaldo Sunkel exportaron pensamiento económico, como A. G. Frank, C. Sempat A. o E. Faletto exportaban historiografía económica o interpretación económica de la historia; como Theotonio dos Santos teoría social y Paulo Freire teoría pedagógica. El conjunto de producción contribuyó a prestigiar el medio chileno.

2. La abundancia de exportaciones eidéticas desde Chile debe explicarse igualmente por la presencia o utilización de "nuevas tecnologías". En los años 1950s, aparecieron en América latina las ciencias económico-sociales, siendo la CEPAL la principal organización que produjo esta renovación, aunque debe considerarse también al ISEB, la FLACSO y el CLAPCS, entre otros. Se creó una nueva "tecnología" para interpretar la realidad que se transformó en alternativa a las antiguas: pensamiento político y ensayo. Esta nueva tecnología, que adquirió inmensa aceptación, se elaboró para América latina de manera importante en Chile (aunque también en otras partes del Conosur: Buenos Aires y sur de Brasil ${ }^{25}$ ) y en Chile se

25 Este mismo criterio debe servir para entender la hegemonía intelectual paulista, a partir de los 60s. Estas nuevas tecnologías vienen en el marco del marxismo y del estructuralismo. En Sao Paulo se produce la renovación más radical respecto de la generación de los años 20s, que había producido grandes obras durante los 30s: Gilberto Freyre, C. Prado Junior, S. Buarque de Holanda, Fernando de Azevedo. Son los profesores franceses traídos a la U.S.P (María Antonia) quienes conducen a la nueva generación 
utilizó también sistemáticamente para agregar otros discursos como el pensamiento político y el discurso teológico, por ejemplo. Este proceso otorgó a la producción eidética chilena una ventaja respecto de otros exportadores, en relación a Argentina y Brasil, pues allí fue parcialmente inhibida por las dictaduras, o respecto de México o Cuba que asumieron estas nuevas tecnologías o más tarde o débilmente.

3. El aumento y el mejoramiento de las redes fue otra razón. Numerosas personas entre éstas eran extranjeras, gozaban de cierto prestigio antes de instalarse en Chile y se encontraban insertas en redes a través de las cuales importaron y exportaron ideas, es el caso de Prebisch, F. H. Cardoso o R. Vekemans, cuyo trabajo les ayudó a acrecentar tales redes. Otras personas hicieron su prestigio en Chile utilizando redes existentes pero acrecentándolas y aceitándolas como Paulo Freire ${ }^{26}$ y antes Celso Furtado.

Por cierto, el interés por Chile había motivado a redes internacionales a conectarse con Chile, lo que favoreció también la circulación de ideas. Ello ocurrió, como se ha mostrado, con la red Economía y Humanismo y con otras como la neomarxista de Monthly Review y la de economistas del desarrollo. También hubo figuras individuales que conociendo esta producción buscaron acercamientos ${ }^{27}$. Además había otras redes actuantes como la masonería, la III Internacional, la CLACSO que se desarrollaron en el periodo, adquiriendo bandas anchas que permitieran vehiculizar notoriamente más información.

a la adopción de nuevas tecnologías que renuevan (o que crean) la antropología, la sociología, la ciencia política y la economía como disciplinas independientes.

Aunque entre 1955 y 1964, el mayor lugar de creación de estas nuevas tecnologías se encuentra todavía en Río de Janeiro (en el ISEB y el CLAPCS) va a ser, paradójicamente durante los primeros años de la dictadura 1964-1969, que São Paulo lo va a suplantar como polo hegemónico. El líder brasileño de este movimiento es Florestan Fernándes y su discípulo emblemático es F. H. Cardoso.

26 Paulo Freire cuenta en Aprendiendo con la propia historia (1987) como durante los 5 años que estuvo en Chile desarrolló no sólo contactos con chilenos y con brasileños exiliados, sino que también con México y USA, particularmente a través de Iván Illich. Gracias a esto fue invitado para México y Estados Unidos. Cuenta igualmente que "cuando iba a México generalmente trabajaba con 100 o 200 personas, todas de América latina” (1987, p. 122).

27 Una figura muy relevante es el economista egipcio-senegalés Samir Amin, quien había conocido desde los años 1950s, mientras hacía su tesis doctoral en Paris la obra de Prebisch, y durante los 1960s la obra de A. G. Frank, presumiblemente a través de las redes neomarxistas. Amin se interesó en tomar contacto con los latinoamericanos buscando ideas para interpretar la realidad africana y el Tercer Mundo en general. Así, fue uno de los fundadores junto al pakistaní Mahbub Ul Haq y a Enrique Iglesias del Foro Tercer Mundo, cuya primera reunión se hizo en Chile en abril de 1973 (véase www.encuentrointelectuallatinoamericano.org). 
4. Esto de las redes debe complementarse con la cuestión de la sinergia. El aumento de la cantidad de redes y la multiplicación de los mensajes en cada una generó una sinergia tal que permitió la acumulación de energía para hacer hervir el pequeño medio nacional. La densidad de producción y de figuras hizo que éste pequeño medio político-intelectual entrara en una suerte de euforia intelectual y política ${ }^{28}$.

La interconexión entre la red cepalino-dependentista con sus agregados o tentáculos: la red ILPES y la red BID (véase Devés, 2000b), la conexión de éstas con la red FLACSO-CLACSO; la conexión con la orden jesuita por un lado, con la Democracia Cristiana latinoamericana y de ésta con la europea y de las dos o tres últimas con Economía y Humanismo y la sociología belga ${ }^{29}$.

5. La política de exportaciones eidéticas en la intelectualidad en Chile durante los largos 60 s trasciende con mucho lo que hicieron los Cristianos por el Socialismo. El trabajo mayor se hizo desde la CEPAL, una parte operó a través de la difusión que hacían las Naciones Unidas de la producción de la Comisión en la medida que ésta formaba parte de aquellas, pero más específica es la difusión del ideario que emprendió la propia CEPAL a través del ILPES (véase ILPES, 1966).

\section{Para terminar}

Seguramente algunos lectores se habrán sorprendido y hasta molestado por este lenguaje económico aplicado a las ideas. Se trata de un intento por explorar una problemática para la cual la eidología no ha elaborado un

28 La densidad de grandes figuras extranjeras, algunas residentes como Prebisch, Freire, Frank, Iglesias y tantas más, otras de paso como H. Camara, S. Amin, así como los chilenos de este ambiente: Anibal Pinto, O. Sunkel y de otro ambiente: Pablo Neruda, N Parra, crearon en Santiago y en Chile una situación anómala.

29 En las redes de sociólogos procedentes de Bélgica es muy importante la figura de François Houtart, que tuvo importancia tanto para el ingreso de ideas hacia América latina como para la exportación desde América latina. Houtart estuvo ligado a Cristianos por el Socialismo y fue importante en la recepción de exiliados y estudiantes. A través de ellos también se exportó el pensamiento cristiano-socialista hacía Europa. 
lenguaje: qué hace que haya ciertas ideas que reciben alta aceptación entre comunidades que no las han elaborado y otras que no las reciben; qué hace que esta aceptación decrezca y desaparezca tan rápido como apareció; qué hace que la producción teórica de un país o de una escuela de pensamiento goce de un boom.

La densidad de grandes figuras extranjeras, algunos residentes como Prebisch, Freire, Frank, Iglesias y tantos otros más, otros de paso como H. Camara, S. Amin, así como los chilenos de este ambiente: P. Neruda, N. Parra, crearon en Santiago y en Chile una situación anómala.

Que el consumo de bienes simbólicos (bienes suntuarios) depende de gran medida de las modas parece algo obvio, pero con esto no hacemos sino desplazar el problema, debe entonces elaborarse una teoría de las modas que explique porqué se suceden de tal modo y con tal duración y no de otra forma.

El boom de las exportaciones eidéticas chilenas, de ciencias económico-sociales y humanas de los largos 60s, no es comparable a periodos anteriores, a los que supera de forma sideral, y tampoco a los posteriores, a los que también supera, aunque no tan considerablemente. Es precisamente esta excepcionalidad de los 1960s, que los hace parcialmente incomparables, la que les constituye en "modelo ideal”, pues si aquello fue posible en algún sentido puede volver a serlo.

El boom de exportaciones fue eminentemente "espontáneo", "natural", no-programado. Por una parte fue la producción oportuna en el momento oportuno y eso le otorgó una aceptación que toda la programación y la propaganda no habría obtenido; por otro lado, si puede decirse de modo geopolítico, la exportación de ideas fue algo "natural" para una intelectualidad a la cual el espacio vital santiaguino y chileno se hizo pequeño; una intelectualidad tan potente que con sólo respirar cortaba las correas de la camisa de fuerza de las fronteras nacionales: las grandes personalidades intelectuales de la época publicaron su obra fuera y en algunos casos (Freire, Cardoso y Faletto, Sunkel y Paz, pero también Neruda y otros) en decenas de ediciones.

Es cierto que también hubo una política de exportaciones, como se ha mostrado, por parte de Cristianos por el Socialismo o por parte de la red cepalino-dependentista, pero se trató de casos específicos, desconectados y de ninguna manera fue parte de una política nacional de promoción de las 
exportaciones eidéticas. En ningún caso existió algo como un Pro-Chile $e^{30}$ para promover las exportaciones eidéticas. La creación de la Comisión Nacional de Ciencia y Tecnología (CONICYT) apuntaba a fomentar el desarrollo de la investigación nacional no a exportarla; la creación de Quimantú se fundamentó en la necesidad de promover una cultura masiva y con fines ideológico políticos, pero en ningún caso como una forma de exportar la producción intelectual chilena. Otros estados han desarrollado una política de traducciones, ediciones y exportación de su producción a nivel mundial, uniendo la exportación de sus ideas a su política internacional, como fue el caso de la China de Mao en los 1960s.

Ahora bien ¿Qué podemos extraer de todo esto para potenciar en la actualidad el trabajo intelectual coordinado en el Conosur y América latina? ¿En que sentido la comprensión de un caso de boom de exportaciones eidéticas puede contribuir a hacernos a entender mejor lo que podría ser la circulación de las ideas, en una situación de colaboración intelectual abundante y fluida? Creo que para responder a estas preguntas deben explorarse los siguiente aspectos: la sinergia que se genera entre gente muy productiva, la coordinación entre diversos agentes intelectuales, la importancia de un espacio que atraiga y mantenga a la intelectualidad, la apertura a las nuevas tecnologías en cada disciplina y el aprovechamiento de la sociedad civil intelectual y de sus redes.

\section{Referências}

AAVV. Cristianos por el Socialismo. Santiago: Nuevo Mundo, 1972.

. Los cristianos y la revolución. Santiago: Quimantú, 1973.

ALDUNATE, José. Derechos humanos, derechos de los pobres. Santiago: Rehue, 1994.

. Crónicas de una iglesia liberadora. Santiago: LOM, 2000.

BERRYMAN, Phillip. Teología de la liberación. México: Siglo XXI, 1989.

30 Pro-Chile es un organismo creado por el Estado de Chile en los años 1970s, con el objetivo de promover las exportaciones, particularmente de rubros no tradicionales. 
BOESAK, Allan. "El resurgir desde el desierto", en: TORRES, Sergio; FABELLA, Virginia. El Evangelio Emergente. La Teología desde el reverso de la historia. Salamanca: Sígueme, 1981.

CASTILLO, Jaime. Democracia y Derechos Humanos. Santiago: ICHEH-Pehuen, 1986.

CHONCHOL, Jacques. "Desarrollo económico y sus consecuencias para la agricultura”, en: VEKEMANS, Roger. La tierra y el hombre. Actas del 4. ${ }^{\circ}$ Congreso Internacional Católico de la Vida Rural. Buenos Aires, 1958.

CRISTIANOS POR EL SOCIALISMO. Documento Final, en: AAVV (1973). Los cristianos y la revolución. Santiago: Quimantú, 1972.

CORREA, Sofía. Con las riendas del poder, la derecha chilena en el Siglo XX. Santiago: Sudamericana, 2005.

DEVÉS-VALDÉS, Eduardo. "La CEPAL: red Internacional, actor internacional y promotora de la integración”. Sociologías, Brasil: U. Federal Rio Grande do Sul, año II, n. 3, enero-junio, 2000a.

. El Pensamiento Latinoamericano en el Siglo XX. Entre la modernización y la identidad. Tomo I, Del Ariel de Rodó a la Cepal (1900-1950). Buenos AiresSantiago: Biblos-DIBAM, 2000b.

. “Cómo pasaron las ideas socioeconómicas Latinoamericanas a África anglófona entre 1960-1980? Retransmisores de ideas Latinoamericanas: Dudley Seers y el Institut of Development Studies". Latinoamérica. Revista de Estudios Latinoamericanos, n. 39, México: CCYDEL-UNAM, 2004.

"Recepción y reelaboración del pensamiento económico-social chileno y latinoamericano en Tanzania 1965-1985: Su proceso de africanización”. Atenea, n. 492, Chile: Universidad de Concepción, p. 45-68, 2005.

. Senegal, "La reelaboración de las ciencias económico-sociales en África: el caso de Senegal en el marco de las redes intelectuales al sur del Sahara, (19651985)"'. Revista Estudos Afroasiaticos, Rio de Janeiro: U. Cândido Mendes, Río de Janeiro, primer semestre 2006.

DIAZ-NIEVA, José. Chile: de la Falange Nacional a la Democracia Cristiana. Madrid: UNED, 2001.

FARIÑA, Carmen. "Notas sobre el pensamiento corporativo de la juventud conservadora a través del periódico Lircay (1934-1940)". Revista de Ciencia Política, v. IX, n. 1, Santiago: Universidad Católica, 1987.

FREI, Eduardo. La política y el espíritu. Santiago: Ercilla, 1940. 
. Memorias 1911-1934. Santiago: Planeta, 1989.

FREIRE, Paulo. Aprendendo com a própria história. Río de Janeiro: Paz e Terra, 1978.

ILPES. Instituto Latinoamericano de Planificación Económica y Social. Santiago: ILPES, 1966.

LEBRET, L. J. Suicídio ou sobrevivência do Ocidente? 4. ed. São Paulo: Duas Cidades, 1964.

MALLEY, François. El padre Lebret. La economía a servicio del hombre. Buenos Aires: Carlos Lohle, 1969.

MISTRAL, Gabriela. Escritos políticos de Gabriela Mistral. Editados por Jaime Quezada, F. C. E., Santiago, 1994.

MOREAU, León José. “Economía y Humanismo”. Política y Espíritu, n. 60, año VII, Santiago, julio 1951.

MORENO-VALENCIA, Fernando. Antecedentes históricos y estructura de la teología de la liberación. Santiago: Instituto de Ciencia Política de la Universidad de Chile, 1985.

MOULIAN, Tomás. La Democracia Cristiana en su fase ascendente: 1957-1964. Documento 288. Santiago: FLACSO, 1986.

PINEDO, Javier. "El pensamiento de los ensayistas y cientistas sociales en los largos años 60 en Chile (1958-1973). Los herederos de Francisco A. Encina”. Atenea, Santiago: U. de Concepción, segundo semestre 2005.

PROTOPAPAS, Jorge. Los cristianos por el cambio en América Latina. Santiago: Pacífico, 1974.

QUOIST, Michel. "Epílogo" a FUSEAU, Jean-Pierre. Odile Simon. Paris: Les Éditions Ouvriéres, s/f.

RUIZ, Carlos; CRISTI, Renato. El pensamiento conservador en Chile. Santiago: Universitaria, 1992.

SCHILlING, Paulo. "Prólogo" a CÂMARA, Helder. Escritos. Buenos Aires: Schapire, 1972.

SERRANO, Sol. "Del conservadurismo a la modernización: la visión histórica de la Falange Nacional de Chile". Alternativas. Santiago: Centro de Estudios de la Realidad Contemporánea, 1984.

SILVA-SOLAR, Julio; CHONCHOL, Jacques. ¿Qué es el socialcristianismo? Ensayo de interpretación. Santiago: Caja Hogar San Pancracio, 1948. 
TORRES, Sergio; EAGLESON, John. Teología de las Américas. Salamanca: Sigueme, 1980.

VEKEMANS, Roger. Marginalidad: incorporación e integración. Santiago: DESAL, 1967.

. D.C.-C.I.A.-CELAM. Autopsia del mito Vekemans. Santiago: U. Católica de Tachira, 1982.

ZEA, Leopoldo. El pensamiento latinoamericano. Barcelona: Ariel, 1976.

Recebido em abril de 2010.

Aprovado em abril de 2010. 